\title{
Research Article \\ Geometry of Hamiltonian Dynamics with Conformal Eisenhart Metric
}

\author{
Linyu Peng, ${ }^{1}$ Huafei Sun, ${ }^{2}$ and Xiao Sun ${ }^{3}$ \\ ${ }^{1}$ Department of Mathematics, University of Surrey, Guildford GU2 7XH, UK \\ ${ }^{2}$ Department of Mathematics, Beijing Institute of Technology, Beijing 100081, China \\ ${ }^{3}$ School of Automation, Beijing Institute of Technology, Beijing 100081, China \\ Correspondence should be addressed to Huafei Sun, huaf.sun@gmail.com
}

Received 3 December 2010; Revised 19 March 2011; Accepted 12 April 2011

Academic Editor: Heinrich Begehr

Copyright (C 2011 Linyu Peng et al. This is an open access article distributed under the Creative Commons Attribution License, which permits unrestricted use, distribution, and reproduction in any medium, provided the original work is properly cited.

\begin{abstract}
We characterize the geometry of the Hamiltonian dynamics with a conformal metric. After investigating the Eisenhart metric, we study the corresponding conformal metric and obtain the geometric structure of the classical Hamiltonian dynamics. Furthermore, the equations for the conformal geodesics, for the Jacobi field along the geodesics, and the equations for a certain flow constrained in a family of conformal equivalent nondegenerate metrics are obtained. At last the conformal curvatures, the geodesic equations, the Jacobi equations, and the equations for the flow of the famous models, an $N$ degrees of freedom linear Hamiltonian system and the HénonHeiles model are given, and in a special case, numerical solutions of the conformal geodesics, the generalized momenta, and the Jacobi field along the geodesics of the Hénon-Heiles model are obtained. And the numerical results for the Hénon-Heiles model show us the instability of the associated geodesic spreads.
\end{abstract}

\section{Introduction}

As the development of differential geometry, symplectic geometry, and Riemannian geometry, the field of dynamics has been studied from the point of geometry, and many significant results have been reached, especially in Hamiltonian dynamics. In order to consider the stability of the dynamical systems, it is usually necessary and efficient for one to study the geometric structure of them.

In $[1,2]$, the applications of methods used in classical differential geometry are concerned to study the chaotic dynamics of Hamiltonian systems. After getting the geodesics in the configuration space which is equipped with a suitable metric, the geometry theory of chaotic dynamics is investigated and applied into studying the Kepler problem and 
the restricted three body problem. Iwai and Yamaoka [3] proposed a problem, that is how a many-body system behaves in a neighborhood of a collinear configuration and dealt with the behavior of boundaries for three bodies in space. From the viewpoint of Riemannian geometry, similarly to the method for obtaining Jacobi equation for geodesic deviations, the equations of the variational vector obtained along the solution of the equations of motion, are used to study the boundary behavior, and small vibrations near an equilibrium of dynamical systems. Moreover, geometry is also applied to other fields of dynamics, such as Hamiltonian and gradient control system [4], nonlocal Hamiltonian operators [5], hydrodynamics and realization [6], fluid mechanics [7], quantum systems [8], reaction dynamics [9], and other dynamics systems [10-12].

In the present paper, in Section 2, the foundation of the Hamiltonian mechanics, the Eisenhart metric of a manifold $M \times \mathbb{R}^{2}$, where $M$ is the configuration space, and the corresponding geometric structure are introduced. We study the conformal Eisenhart metric in Section 3, and the curvatures, the geodesic equations, the equations of Jacobi field along the geodesics, and the equations of a certain flow for the classical Hamiltonian dynamics are obtained. Moreover, in Section 4, the conformal geometric structures of two models are given, and numerical simulations for one of the models are shown. The final Section 5 is devoted to the conclusion.

\section{Geometry and Dynamics}

\subsection{Geometry and Flow}

To make the paper readable, we recall some useful background of Riemannian geometry. The readers could refer to [13-15] for more information. Let $\left(M^{N}, g, D\right)$ be a smooth manifold with dimension $N$, where $g$ is a nondegenerate metric defined on the vector field $\mathfrak{X}(M)$, and $D$ is the affine connection, which is defined as

$$
D_{X} Y=X^{j}\left(\frac{\partial Y^{i}}{\partial x^{j}}+\Gamma_{j k}^{i} Y^{k}\right) \frac{\partial}{\partial x_{i}}, \quad \forall X, Y \in \mathfrak{X}(M)
$$

The coefficients $\Gamma_{i j}^{k}$ of the connection $D$ can be represented in terms of the metric tensor $g$ by

$$
\Gamma_{i j}^{k}=\frac{1}{2} g^{k l}\left(\partial_{j} g_{i l}+\partial_{i} g_{j l}-\partial_{l} g_{i j}\right)
$$

where $\partial_{i}$ means $\partial / \partial x^{i}$, and the Einstein summation convention is used. For any $X, Y, Z \in$ $\mathfrak{X}(M)$, the curvature tensor is defined by

$$
R(X, Y) Z=D_{X} D_{Y} Z-D_{Y} D_{X} Z-D_{[X, Y]} Z,
$$

where $[\cdot, \cdot]$ is the Lie bracket on $\mathfrak{X}(M)$ defined as $[X, Y]=X Y-Y X$. Then the components of the curvature tensor can be given by

$$
R_{k i j}^{l}=\partial_{i} \Gamma_{k j}^{l}-\partial_{j} \Gamma_{k i}^{l}+\Gamma_{k j}^{h} \Gamma_{h i}^{l}-\Gamma_{k i}^{h} \Gamma_{h j}^{l} .
$$


The Ricci curvature is defined by

$$
R_{i j}=g^{k l} R_{i k l j}
$$

where $R_{i k l j}=g_{k s} R_{i l j}^{s}$. The scalar curvature is defined by

$$
R=g^{i j} R_{i j}
$$

A curve $\xi(t)$ on $M$ is said to be geodesic if its tangent $\dot{\xi}(t)$ is displaced parallel along the curve $\xi(t)$, that is,

$$
D_{\dot{\xi}(t)} \dot{\xi}(t)=0
$$

In local coordinate $\xi(t)=\left(x^{1}(t), x^{2}(t), \ldots, x^{N}(t)\right)$, the geodesic equation satisfies

$$
\frac{\mathrm{d}^{2} x^{k}}{\mathrm{~d} t^{2}}+\Gamma_{i j}^{k} \frac{\mathrm{d} x^{i}}{\mathrm{~d} t} \frac{\mathrm{d} x^{j}}{\mathrm{~d} t}=0
$$

In addition, the equation for the well-known Jacobi field $J=J^{k} \partial_{k}$ satisfies

$$
D_{\dot{\xi}(t)} D_{\dot{\xi}(t)} J=R(\dot{\xi}(t), J) \dot{\xi}(t),
$$

where $\xi(t)$ is a geodesic on manifold $M$. It is also called the geodesic derivation equation, as its close connection with the behavior or completeness of the geodesics. Usually Jacobi field is used to study the stability of the geodesic spreads, that is, the behavior of the geodesics with the time parameter $t$ changing, of dynamical systems. In [16], the instability of the geodesic spreads of the entropic dynamical models is obtained via the study of the Jacobi field. The readers could also refer to [17] for more about its applications to physical systems.

A metric $\tilde{g}$ defined on $M$ is said to be conformally equivalent to $g$, if there exists a function $f \in C^{\infty}(M)$ such that

$$
\tilde{g}=e^{f} g
$$

Then the equivalent relation between metrics and the equivalence class of $g$ is called its conformal class denoted by $[g]$. In local coordinate, (2.10) becomes

$$
\tilde{g}_{i j}=e^{f} g_{i j}
$$


Hamilton [18] introduced the Ricci flow in 1982, which ultimately led to the proof, by Perelman, of the Thurston geometrization conjecture and the solution of the Poincare conjecture. It is a geometric evolution equation in which one starts with a smooth Riemannian manifold $\left(M^{N}, g_{0}\right)$ and evolves its metric by the equation

$$
\left(\frac{\partial g_{t}}{\partial t}\right)_{i j}=-2 R_{i j}
$$

where $\left.g_{t}\right|_{t=0}=g_{0}$ and $R_{i j}$ denote the Ricci curvatures with $g_{t}$.

In this paper, we study a similar flow, which is an evolution equation started from a smooth manifold $M^{N}$ with nondegenerate metric $g$, which is not needed to be Riemannian.

\subsection{Geometry in Hamiltonian Dynamics}

In mechanics [19], a Lagrangian function $L$ of a dynamical system of $N$ degrees of freedom is usually defined by $L\left(q^{i}, \dot{q}^{i}, t\right)$, where $q=\left(q^{1}, \ldots, q^{N}\right)$ and $\dot{q}=\left(\dot{q}^{1}, \ldots, \dot{q}^{N}\right)$ are called the generalized coordinate and generalized velocity, respectively. While a Hamiltonian function is represented as $H\left(q^{i}, p_{i}, t\right)$, where $p=\left(\partial L / \partial q^{1}, \ldots, \partial L / \partial q^{N}\right)$ is generalized momenta. The space $M$ of the generalized coordinate is called the configuration space.

The Hamiltonian action within $t \in[a, b]$ is

$$
S=\int_{a}^{b} L(q, \dot{q}, t) \mathrm{d} t
$$

The Hamiltonian variational principle states

$$
\delta S=0 .
$$

From the calculus of variation, (2.14) equates to the Euler-Lagrange equations

$$
\frac{\mathrm{d}}{\mathrm{d} t}\left(\frac{\partial L}{\partial \dot{q}^{i}}\right)-\frac{\partial L}{\partial q^{i}}=0, \quad i=1, \ldots, N
$$

To pass to the Hamiltonian formalism, we introduce the generalized momenta as

$$
p_{i}=\frac{\partial L}{\partial \dot{q}^{i}}, \quad i=1, \ldots, N,
$$


make the change $\left(q^{i}, \dot{q}^{i}\right) \mapsto\left(q^{i}, p_{i}\right)$, and introduce the Hamiltonian

$$
H\left(q^{i}, p_{i}, t\right)=p_{j} \dot{q}^{j}-L\left(q^{i}, \dot{q}^{i}, t\right)
$$

Then the Euler-Lagrange equations are equivalent to the Hamiltonian equations, which are given by

$$
\frac{\mathrm{d} q^{i}}{\mathrm{~d} t}=\frac{\partial H}{\partial p_{i}}, \quad \frac{\mathrm{d} p_{i}}{\mathrm{~d} t}=-\frac{\partial H}{\partial q^{i}}, \quad i=1, \ldots, N
$$

In this paper, we will only consider the classical Hamiltonian dynamics, whose Hamiltonian is of the form

$$
H=\frac{1}{2} \sum_{i=1}^{N} p_{i}^{2}+V(q)
$$

where $V(q)$ is the potential energy.

The energy $E$ of the classical Hamiltonian dynamics is a constant equal to Hamiltonian $H$. Considering the manifold $M \times \mathbb{R}^{2}$ defined by

$$
M \times \mathbb{R}^{2}=\left\{q \mid q=\left(q^{0}, q^{1}, q^{2}, \ldots, q^{N}, q^{N+1}\right)\right\}
$$

in which $q^{0}=t$ and $q^{N+1}=\left(C_{1}^{2} / 2\right) t+C_{2}^{2}-\int_{0}^{t} L \mathrm{~d} \tau$, where $C_{1}, C_{2}$ are real numbers, and in the following, $C_{1}$ is assumed to be equal to 1 .

In this paper, we assume that, the Greek symbols $\alpha, \beta, \gamma, \ldots$ are from 0 to $N+1$ and the Latin symbols $i, j, k, \ldots$ are from 1 to $N$.

The Eisenhart metric (cf. [20]) of the manifold $M \times \mathbb{R}^{2}$ is defined as

$$
\left(g_{\alpha \beta}\right)=\left(\begin{array}{ccccc}
-2 V & 0 & \cdots & 0 & 1 \\
0 & & & & 0 \\
\vdots & & I_{N \times N} & \vdots \\
0 & & & & 0 \\
1 & 0 & \cdots & 0 & 0
\end{array}\right),
$$

where $I_{N \times N}$ is the identity matrix, and we know that it is not $\operatorname{degenerate}$ as $\operatorname{det}\left(g_{\alpha \beta}\right)=$ $(-1)^{N+3}$. 
The inverse matrix of $\left(g_{\alpha \beta}\right)$ is

$$
\left(g^{\alpha \beta}\right)=\left(\begin{array}{ccccc}
0 & 0 & \cdots & 0 & 1 \\
0 & & & 0 \\
\vdots & I_{N \times N} & \vdots \\
0 & & & 0 \\
1 & 0 & \cdots & 0 & 2 V
\end{array}\right) .
$$

The nonzero coefficients of the Riemanian connection are

$$
\Gamma_{00}^{k}=-\Gamma_{0 k}^{N+1}=\partial_{k} V
$$

where $\partial_{k}$ means $\partial / \partial q^{k}$.

The nonzero components of the curvature tensor are

$$
R_{0 j 0 l}=-\partial_{j} \partial_{l} V
$$

The nonzero Ricci curvature and the scalar curvature are, respectively, given by

$$
\begin{gathered}
R_{00}=\Delta V, \\
\mathcal{R}=0,
\end{gathered}
$$

where $\Delta$ is the Laplacian operator in the Euclidean space.

The geodesic equations read

$$
\begin{gathered}
\frac{\mathrm{d}^{2} q^{0}}{\mathrm{~d} t^{2}}=0 \\
\frac{\mathrm{d}^{2} q^{i}}{\mathrm{~d} t^{2}}=-\frac{\partial V}{\partial q^{i}}, \quad i=1,2, \ldots N, \\
\frac{\mathrm{d}^{2} q^{N+1}}{\mathrm{~d} t^{2}}=-\frac{\mathrm{d} L}{\mathrm{~d} t}
\end{gathered}
$$

in which, the first and third equations are identical, and the second ones are the equations of motion for the associated dynamics.

\section{Conformal Structure}

From now on, in order to study the conformal structure of the manifold $M \times \mathbb{R}^{2}$, in which $M$ is the configuration space, we investigate the conformal Eisenhart metric which states as

$$
\tilde{g}=e^{f} g
$$


where $g$ is the Eisenhart metric and $f: M \times \mathbb{R}^{2} \rightarrow \mathbb{R}$ is a smooth function. Then the arc length parameter $\tilde{s}$ is shown as

$$
\begin{aligned}
\mathrm{d} \widetilde{s}^{2} & =\widetilde{g}_{\alpha \beta} \mathrm{d} q^{\alpha} \mathrm{d} q^{\beta} \\
& =C_{1}^{2} e^{f} \mathrm{~d} t^{2} \\
& =e^{f} \mathrm{~d} t^{2} .
\end{aligned}
$$

And the volume element $\mathrm{d} \widetilde{v}$ of the manifold under the conformal Eisenhart metric is given by

$$
\begin{aligned}
\mathrm{d} \tilde{v} & =\sqrt{\operatorname{det}(\tilde{g})} \mathrm{d} q^{0} \wedge \mathrm{d} q^{1} \wedge \cdots \wedge \mathrm{d} q^{N+1} \\
& =-e^{((N / 2)+1) f} \mathrm{~d} q^{0} \wedge \mathrm{d} q^{1} \wedge \cdots \wedge \mathrm{d} q^{N+1}
\end{aligned}
$$

Proposition 3.1. The conformal and independent components of the curvature tensor are given by

$$
\begin{aligned}
\widetilde{R}_{0 j 0 l}= & e^{f}\left(-\partial_{j} \partial_{l} V-V \partial_{j} \partial_{l} f+\frac{1}{2} V \partial_{j} f \partial_{l} f\right) \\
& +\delta_{j l} \frac{e^{f}}{4}\left(2 \partial_{0} \partial_{0} f-\left(\partial_{0} f\right)^{2}-4 V \partial_{0} f \partial_{N+1} f-4 V^{2}\left(\partial_{N+1} f\right)^{2}-2(\nabla f \cdot \nabla V)\right. \\
& \left.-2 V|\nabla f|^{2}\right), \\
\widetilde{R}_{0 j 0 N+1}= & e^{f}\left(-\frac{1}{2} \partial_{0} \partial_{j} f-V \partial_{j} \partial_{N+1} f+\frac{1}{4} \partial_{0} f \partial_{j} f+\frac{1}{2} V \partial_{j} f \partial_{N+1} f-\frac{1}{2} \partial_{j} V \partial_{N+1} f\right), \\
\widetilde{R}_{0 j k l}= & \delta_{j l} e^{f}\left(\frac{1}{2} \partial_{0} \partial_{k} f+\frac{1}{2} \partial_{k} V \partial_{N+1} f-\frac{1}{4} \partial_{0} f \partial_{k} f\right) \\
& -\delta_{j k} e^{f}\left(\frac{1}{2} \partial_{0} \partial_{l} f+\frac{1}{2} \partial_{l} V \partial_{N+1} f-\frac{1}{4} \partial_{0} f \partial_{l} f\right) \\
\widetilde{R}_{0 j k N+1}= & e^{f}\left(-\frac{1}{2} \partial_{j} \partial_{k} f+\frac{1}{4} \partial_{j} f \partial_{k} f\right) \\
& -\delta_{j k} e^{f}\left(\frac{1}{2} \partial_{0} \partial_{N+1} f+\frac{1}{2} V\left(\partial_{N+1} f\right)^{2}+\frac{1}{4} \partial_{0} f \partial_{N+1} f+\frac{1}{4}|\nabla f|^{2}\right)
\end{aligned}
$$




$$
\begin{aligned}
\tilde{R}_{0 N+10 N+1}= & -e^{f}\left(\partial_{0} \partial_{N+1} f-V \partial_{N+1} \partial_{N+1} f+\frac{1}{4}|\nabla f|^{2}\right), \\
\tilde{R}_{0 N+1 k l}= & 0, \\
\tilde{R}_{0 N+1 k N+1}= & e^{f}\left(-\frac{1}{2} \partial_{k} \partial_{N+1} f+\frac{1}{4} \partial_{k} f \partial_{N+1} f\right), \\
\widetilde{R}_{i j k l}= & \delta_{j l} e^{f}\left(\frac{1}{2} \partial_{i} \partial_{k} f-\frac{1}{4} \partial_{i} f \partial_{k} f\right)-\delta_{i l} e^{f}\left(\frac{1}{2} \partial_{j} \partial_{k} f-\frac{1}{4} \partial_{j} f \partial_{k} f\right) \\
& +\delta_{i k} e^{f}\left(\frac{1}{2} \partial_{j} \partial_{l} f-\frac{1}{4} \partial_{j} f \partial_{l} f\right)-\delta_{j k} e^{f}\left(\frac{1}{2} \partial_{i} \partial_{l} f-\frac{1}{4} \partial_{i} f \partial_{l} f\right) \\
& +\left(\delta_{i k} \delta_{j l}-\delta_{i l} \delta_{j k}\right) e^{f}\left(\frac{1}{2} \partial_{0} f \partial_{N+1} f+\frac{1}{2} V\left(\partial_{N+1} f\right)^{2}+\frac{1}{4}|\nabla f|^{2}\right), \\
\tilde{R}_{i j k N+1}= & \delta_{i k} e^{f}\left(\frac{1}{2} \partial_{j} \partial_{N+1} f-\frac{1}{4} \partial_{j} f \partial_{N+1} f\right)-\delta_{j k} e^{f}\left(\frac{1}{2} \partial_{i} \partial_{N+1} f-\frac{1}{4} \partial_{i} f \partial_{N+1} f\right), \\
\widetilde{R}_{i N+1 k N+1}= & \delta_{i k} e^{f}\left(\frac{1}{2} \partial_{N+1} \partial_{N+1} f-\frac{1}{4}\left(\partial_{N+1} f\right)^{2}\right),
\end{aligned}
$$

where $\nabla$ and $(, \cdot$,$) are the gradient operator and the inner product in the Euclidean space, respectively.$ Proof. From (2.2), one can get the independent conformal coefficients

$$
\begin{aligned}
& \tilde{\Gamma}_{00}^{0}=\partial_{0} f+V \partial_{N+1} f, \quad \tilde{\Gamma}_{00}^{k}=\partial_{k} V+V \partial_{k} f, \\
& \widetilde{\Gamma}_{00}^{N+1}=V \partial_{0} f+2 V^{2} \partial_{N+1} f, \quad \widetilde{\Gamma}_{0 j}^{0}=\frac{1}{2} \partial_{j} f, \\
& \tilde{\Gamma}_{0 j}^{k}=\frac{1}{2} \delta_{j k} \partial_{0} f, \quad \tilde{\Gamma}_{0 j}^{N+1}=-\partial_{j} V, \\
& \tilde{\Gamma}_{0 N+1}^{0}=0, \quad \tilde{\Gamma}_{0 N+1}^{k}=-\frac{1}{2} \partial_{k} f, \quad \widetilde{\Gamma}_{0 N+1}^{N+1}=-V \partial_{N+1} f, \\
& \widetilde{\Gamma}_{i j}^{0}=-\frac{1}{2} \delta_{i j} \partial_{N+1} f, \quad \tilde{\Gamma}_{i j}^{k}=\frac{1}{2} \delta_{i k} \partial_{j} f+\frac{1}{2} \delta_{j k} \partial_{i} f-\frac{1}{2} \delta_{i j} \partial_{k} f, \\
& \widetilde{\Gamma}_{i j}^{N+1}=-\frac{1}{2} \delta_{i j} \partial_{0} f-\delta_{i j} V \partial_{N+1} f, \quad \widetilde{\Gamma}_{i N+1}^{0}=0, \\
& \tilde{\Gamma}_{i N+1}^{k}=\frac{1}{2} \delta_{i k} \partial_{N+1} f, \quad \tilde{\Gamma}_{i N+1}^{N+1}=\frac{1}{2} \partial_{i} f, \quad \tilde{\Gamma}_{N+1 N+1}^{0}=0, \\
& \widetilde{\Gamma}_{N+1 N+1}^{k}=0, \quad \widetilde{\Gamma}_{N+1 N+1}^{N+1}=\partial_{N+1} f .
\end{aligned}
$$

Then from (2.4) and $\widetilde{R}_{\alpha \beta \gamma \eta}=\tilde{g}_{\beta \lambda} \widetilde{R}_{\alpha \gamma \eta^{\prime}}^{\lambda}$ one can get the conclusion, immediately. 
Proposition 3.2. The conformal Ricci curvatures of $M \times \mathbb{R}^{2}$ are given by

$$
\begin{aligned}
\widetilde{R}_{00}= & \Delta V+V \Delta f-\frac{N}{2} \partial_{0} \partial_{0} f+\frac{N}{4}\left(\partial_{0} f\right)^{2}+N V \partial_{0} f \partial_{N+1} f+N V^{2}\left(\partial_{N+1} f\right)^{2} \\
& +\frac{N}{2}(\nabla f \cdot \nabla V)+\frac{N}{2} V|\nabla f|^{2}+2 V \partial_{0} \partial_{N+1} f+2 V^{2} \partial_{N+1} \partial_{N+1} f, \\
\widetilde{R}_{0 k}= & -N\left(\frac{1}{2} \partial_{0} \partial_{k} f+\frac{1}{2} \partial_{k} V \partial_{N+1} f-\frac{1}{4} \partial_{0} f \partial_{k} f\right), \\
\widetilde{R}_{0 N+1}= & -\frac{1}{2} \Delta f-\frac{N+2}{2} \partial_{0} \partial_{N+1} f-V \partial_{N+1} \partial_{N+1} f \\
& -N\left(\frac{1}{2} V\left(\partial_{N+1} f\right)^{2}+\frac{1}{4} \partial_{0} f \partial_{N+1} f+\frac{1}{4}|\nabla f|^{2}\right), \\
\widetilde{R}_{i k}= & -\frac{N}{2} \partial_{i} \partial_{k} f+\frac{N}{4} \partial_{i} f \partial_{k} f \\
& -\delta_{i k}\left(\partial_{0} \partial_{N+1} f+\frac{N}{2} \partial_{0} f \partial_{N+1} f+\frac{N}{2} V\left(\partial_{N+1} f\right)^{2}+\frac{N}{4}|\nabla f|^{2}+V \partial_{N+1} \partial_{N+1} f+\frac{1}{2} \Delta f\right), \\
\widetilde{R}_{i N+1}= & -\frac{N}{2} \partial_{i} \partial_{N+1} f+\frac{N}{4} \partial_{i} f \partial_{N+1} f, \\
\widetilde{R}_{N+1 N+1}= & -\frac{N}{2} \partial_{N+1} \partial_{N+1} f+\frac{N}{4}\left(\partial_{N+1} f\right)^{2} .
\end{aligned}
$$

Proof. From (2.5), and the conformal curvature tensors obtained in Proposition 3.1, we can get the conclusion, immediately.

Theorem 3.3. The conformal scalar curvature is given by

$$
\begin{aligned}
\tilde{\mathcal{R}}= & -e^{-f}\left((N+1) \Delta f+\frac{N(N+1)}{4}|\nabla f|^{2}+2(N+1) \partial_{0} \partial_{N+1} f\right) \\
& -e^{-f}\left(\frac{N(N+1)}{2} \partial_{0} f \partial_{N+1} f+2(N+1) V \partial_{N+1} \partial_{N+1} f+\frac{N(N+1)}{2}\left(\partial_{N+1} f\right)^{2}\right) .
\end{aligned}
$$

Proof. From (2.6), and the conclusion in Proposition 3.2, we can obtain the conclusion of Theorem 3.3, immediately. 
Remark 3.4. When $V$ is a smooth function on the configuration space and let $f=V$, we can get

$$
\begin{gathered}
\tilde{R}_{00}=(V+1)\left(\Delta V+\frac{N}{2}|\nabla V|^{2}\right), \quad \tilde{R}_{0 k}=0, \quad \tilde{R}_{0 N+1}=-\frac{1}{2} \Delta V-\frac{N}{4}|\nabla V|^{2}, \\
\tilde{R}_{i k}=-\frac{N}{2} \partial_{i} \partial_{k} V+\frac{N}{4} \partial_{i} V \partial_{k} V-\delta_{i k}\left(\frac{1}{2} \Delta V+\frac{N}{4}|\nabla V|^{2}\right), \\
\widetilde{R}_{i N+1}=0, \quad \widetilde{R}_{N+1 N+1}=0, \\
\tilde{R}=-e^{-V}\left((N+1) \Delta V+\frac{N(N+1)}{4}|\nabla V|^{2}\right) .
\end{gathered}
$$

Theorem 3.5. The conformal geodesic equations are

$$
\frac{\mathrm{d}^{2} q^{k}}{\mathrm{~d} t^{2}}+\frac{6 E-4 V-1}{2(1-2 L)} \partial_{N+1} f \frac{d q^{k}}{d t}-\frac{1}{2} \partial_{k} f\left(\frac{\mathrm{d} q^{k}}{\mathrm{~d} t}\right)^{2}+\left(E-V+\frac{1}{2}\right) \partial_{k} f+\partial_{k} V=0
$$

and $f$ satisfies

$$
\begin{gathered}
\partial_{0} f=L \partial_{N+1} f, \\
\left(\frac{1}{2}-L\right) \frac{\mathrm{d} f}{\mathrm{~d} t}=2\left(E-V-\frac{1}{4}\right) \partial_{N+1} f,
\end{gathered}
$$

where

$$
\begin{gathered}
\frac{\mathrm{d} f}{\mathrm{~d} t}=\partial_{i} f \frac{\mathrm{d} q^{i}}{\mathrm{~d} t}, \quad i=1, \ldots, N \\
L=E-2 V .
\end{gathered}
$$

Proof. From the geodesic equation (2.8), and the coefficients of Riemannian connection in (3.5), we can get the equations of Theorem 3.5.

For the classical Hamiltonian system, $L$ is usually not a constant. Then if $f$ is constrained in $C^{\infty}(M)$, the second equation in (3.10) can be reduced into $\mathrm{d} f / \mathrm{d} t=0$. Therefore, we can get the following.

Remark 3.6. When assuming $f: M \rightarrow \mathbb{R}$, the geodesic equations are

$$
\frac{\mathrm{d}^{2} q^{k}}{\mathrm{~d} t^{2}}-\frac{1}{2} \partial_{k} f\left(\frac{\mathrm{d} q^{k}}{\mathrm{~d} t}\right)^{2}+\left(E-V+\frac{1}{2}\right) \partial_{k} f+\partial_{k} V=0,
$$


and $f$ satisfies that

$$
\frac{\mathrm{d} f}{\mathrm{~d} t}=0
$$

Lemma 3.7 (Hopf). Let $(M, g)$ be a compact manifold, $f \in C^{\infty}(M)$. Then $\int_{M} \Delta_{g} f=0$.

Lemma 3.8. Let $f, h \in C^{\infty}(M), \tilde{g}=e^{f} g$, then

$$
\begin{gathered}
\nabla_{\tilde{g}} h=e^{-f} \nabla_{g} h, \\
\Delta_{\tilde{g}} h=e^{-f} \Delta_{g} h+\frac{N-2}{2} \widetilde{g}\left(\nabla_{\tilde{g}} f, \nabla_{\tilde{g}} h\right) .
\end{gathered}
$$

Proof. From the definition of gradient and Laplacian of $h \in C^{\infty}(M)$, one can obtain

$$
\begin{aligned}
\nabla_{\tilde{g}} h & =\partial_{i} h \tilde{g}^{i j} \partial_{j}=e^{-f} \partial_{i} h g^{i j} \partial_{j} \\
& =e^{-f} \nabla_{g} h, \\
\Delta_{\tilde{g}} h & =\frac{1}{\sqrt{\widetilde{G}}} \partial_{i}\left(\sqrt{\tilde{G}} \widetilde{g}^{i j} \partial_{j} h\right)=e^{-(N / 2) f} \frac{1}{\sqrt{G}} \partial_{i}\left(e^{((N-2) / 2) f} \sqrt{G} g^{i j} \partial_{j} h\right) \\
& =e^{-f} \frac{1}{\sqrt{G}} \partial_{i}\left(\sqrt{G} g^{i j} \partial_{j} h\right)+\frac{N-2}{2} e^{-f} g^{i j} \partial_{j} h \partial_{i} f \\
& =e^{-f} \Delta_{g} h+\frac{N-2}{2} \tilde{g}\left(\nabla_{\tilde{g}} f, \nabla_{\tilde{g}} h\right),
\end{aligned}
$$

where $G=\operatorname{det}\left(g_{i j}\right)$.

Theorem 3.9. Let $f: M \rightarrow \mathbb{R}, M \times U_{1} \times U_{2}$ be compact, where $U_{1}$ and $U_{2}$ are compact subsets of $\mathbb{R}$. Then from Theorem 3.3, Lemmas 3.7 and 3.8, one can get that

$$
\begin{aligned}
\int_{M \times U_{1} \times U_{2}} \widetilde{R} & =-\int_{M \times U_{1} \times U_{2}} e^{-f}\left((N+1) \Delta f+\frac{N(N+1)}{4}|\nabla f|^{2}\right) \\
& =-\int_{M \times U_{1} \times U_{2}}\left((N+1) \Delta_{\tilde{g}} f-\frac{(N+1)(N-4)}{4} e^{-f}|\nabla f|^{2}\right) \\
& =\frac{(N+1)(N-4)}{4} \int_{M \times U_{1} \times U_{2}}\left(e^{-f}|\nabla f|^{2}\right),
\end{aligned}
$$

and moreover,

(1) when $N>4, \int_{M \times U_{1} \times U_{2}} \tilde{R} \geq 0$,

(2) when $N=4, \int_{M \times U_{1} \times U_{2}} \widetilde{R} \equiv 0$,

(3) when $N<4, \int_{M \times U_{1} \times U_{2}} \tilde{R} \leq 0$. 
In addition, when $N \neq 4$ and the equality of (3.16) holds, $\left|\nabla_{\tilde{g}} f\right|^{2}=e^{-2 f}|\nabla f|^{2}=0$, so that $f$ is a constant function on the configuration space $M$.

Theorem 3.10. Let $f: M \rightarrow \mathbb{R}$ be a smooth function. With the conformal Eisenhart metric, the equations for Jacobi field along the geodesics, which is the solution of (3.12), are given by

$$
\begin{gathered}
\frac{\mathrm{d}^{2} J^{0}}{\mathrm{~d} t^{2}}+\partial_{i} f \frac{\mathrm{d} J^{i}}{\mathrm{~d} t}+A_{1} J^{0}+\partial_{i} \partial_{j} f \frac{\mathrm{d} q^{j}}{\mathrm{~d} t} J^{i}=0, \\
\frac{\mathrm{d}^{2} J^{i}}{\mathrm{~d} t^{2}}+A_{2} \frac{\mathrm{d} J^{0}}{\mathrm{~d} t}+A_{3} \frac{\mathrm{d} J^{j}}{\mathrm{~d} t}-\partial_{i} f \frac{\mathrm{d} J^{N+1}}{\mathrm{~d} t}+A_{4} J^{j}=0, \\
\frac{\mathrm{d}^{2} J^{N+1}}{\mathrm{~d} t^{2}}-2 \frac{\mathrm{d} V}{\mathrm{~d} t} \frac{\mathrm{d} J^{0}}{\mathrm{~d} t}+A_{5} \frac{\mathrm{d} J^{j}}{\mathrm{~d} t}+A_{6} J^{0}+A_{7} J^{i}+A_{8} J^{N+1}=0,
\end{gathered}
$$

where

$$
\begin{aligned}
A_{1}= & \frac{1}{2} \partial_{i} f \frac{\mathrm{d}^{2} q^{i}}{\mathrm{~d} t^{2}}+\frac{1}{2}(\nabla f \cdot \nabla V)-\frac{2 L+1}{8}|\nabla f|^{2}, \\
A_{2}= & 2 \partial_{i} V+\left(E-\frac{1}{2}\right) \partial_{i} f, \\
A_{3}= & \partial_{j} f \frac{\mathrm{d} q^{i}}{\mathrm{~d} t}-\partial_{i} f \frac{\mathrm{d} q^{j}}{\mathrm{~d} t}, \\
A_{4}= & \frac{1}{2}\left(\delta_{i k} \partial_{j} f+\delta_{i j} \partial_{k} f-\delta_{k j} \partial_{i} f\right) \frac{\mathrm{d}^{2} q^{k}}{\mathrm{~d} t^{2}}+\partial_{i} \partial_{j} V-\frac{1}{4} \delta_{i j}|\nabla f|^{2} \\
& +\frac{1}{2}\left(\partial_{i} V \partial_{j} f+\partial_{j} V \partial_{i} f-\partial_{i} \partial_{j} f+\delta_{i j}(\nabla f \cdot \nabla V)+\partial_{j} \partial_{s} f \frac{\mathrm{d} q^{s}}{\mathrm{~d} t} \frac{\mathrm{d} q^{j}}{\mathrm{~d} t}\right), \\
A_{5}= & -2 \partial_{j} V+\left(\frac{1}{2}-L\right) \partial_{j} f, \\
A_{6}= & -\partial_{j} V \frac{\mathrm{d}^{2} q^{j}}{\mathrm{~d} t^{2}}+\frac{1}{2}(\nabla f \cdot \nabla V)-|\nabla V|^{2}, \\
A_{7}= & -2 \partial_{i} \partial_{j} V \frac{\mathrm{d} q^{j}}{\mathrm{~d} t}+\left(\frac{1}{2}-L\right) \partial_{i} \partial_{j} f \frac{\mathrm{d} q^{j}}{\mathrm{~d} t}, \\
A_{8}= & \frac{1}{2} \partial_{j} f \frac{\mathrm{d}^{2} q^{j}}{\mathrm{~d} t^{2}}+\frac{1}{2}(\nabla f \cdot \nabla V)-\frac{1}{4}|\nabla f|^{2} .
\end{aligned}
$$

Proof. Firstly, from the Jacobi equation (2.9), we can get the conformal Jacobi equations represented in components as

$$
\frac{\mathrm{d}^{2} J^{\alpha}}{\mathrm{d} t^{2}}+2 \tilde{\Gamma}_{\beta \sigma}^{\alpha} \frac{\mathrm{d} q^{\beta}}{\mathrm{d} t} \frac{\mathrm{d} J^{\sigma}}{\mathrm{d} t}+\left(\tilde{\Gamma}_{\beta \sigma}^{\alpha} \frac{\mathrm{d}^{2} q^{\beta}}{\mathrm{d} t^{2}}+\tilde{\Gamma}_{\beta \sigma}^{\gamma} \tilde{\Gamma}_{\gamma \lambda}^{\alpha} \frac{\mathrm{d} q^{\beta}}{\mathrm{d} t} \frac{\mathrm{d} q^{\lambda}}{\mathrm{d} t}+\partial_{\lambda} \tilde{\Gamma}_{\beta \sigma}^{\alpha} \frac{\mathrm{d} q^{\beta}}{\mathrm{d} t} \frac{\mathrm{d} q^{\lambda}}{\mathrm{d} t}-\tilde{R}_{\beta \lambda \sigma}^{\alpha} \frac{\mathrm{d} q^{\beta}}{\mathrm{d} t} \frac{\mathrm{d} q^{\lambda}}{\mathrm{d} t}\right) J^{\sigma}=0
$$


where $\widetilde{R}_{\beta \curlywedge \sigma}^{\alpha}$ are given by (2.4). From (2.4), (3.19) turns into

$$
\frac{\mathrm{d}^{2} J^{\alpha}}{\mathrm{d} t^{2}}+2 \tilde{\Gamma}_{\beta \sigma}^{\alpha} \frac{\mathrm{d} q^{\beta}}{\mathrm{d} t} \frac{\mathrm{d} J^{\sigma}}{\mathrm{d} t}+\left(\tilde{\Gamma}_{\beta \sigma}^{\alpha} \frac{\mathrm{d}^{2} q^{\beta}}{\mathrm{d} t^{2}}+\widetilde{\Gamma}_{\beta \lambda}^{\gamma} \tilde{\Gamma}_{\gamma \sigma}^{\alpha} \frac{\mathrm{d} q^{\beta}}{\mathrm{d} t} \frac{\mathrm{d} q^{\lambda}}{\mathrm{d} t}+\partial_{\sigma} \widetilde{\Gamma}_{\beta \lambda}^{\alpha} \frac{\mathrm{d} q^{\beta}}{\mathrm{d} t} \frac{\mathrm{d} q^{\lambda}}{\mathrm{d} t}\right) J^{\sigma}=0 .
$$

Furthermore, substituting (3.5) into (3.20), in which $f$ should be constrained in $C^{\infty}(M)$ but not $C^{\infty}\left(M \times \mathbb{R}^{2}\right)$, we complete the proof of Theorem 3.10.

Now let $f_{u}: M \times[a, b] \rightarrow \mathbb{R}$ be a smooth function with $\left.f_{u}\right|_{u=0}=0$, and $g_{u}=e^{f_{u}} g$, that is, $\left.g_{u}\right|_{u=0}=g$, where $g$ is the Eisenhart metric. Then we can get the following.

Theorem 3.11. The equations of the flow defined in (2.12) are

$$
\begin{gathered}
e^{f_{u}} \frac{\partial f_{u}}{\partial u}=-\Delta f_{u}-\frac{N}{2}\left|\nabla f_{u}\right|^{2}, \\
\Delta V+\frac{N}{2}\left(\nabla f_{u} \cdot \nabla V\right)=0, \\
2 \partial_{i} \partial_{k} f_{u}-\partial_{i} f_{u} \partial_{k} f_{u}=0, \quad i \neq k, \\
\Delta f_{u}-N \partial_{i} \partial_{i} f_{u}+\frac{N}{2}\left(\partial_{i} f_{u}\right)^{2}+\frac{N-1}{2}\left|\nabla f_{u}\right|^{2}=0, \quad i=1,2, \ldots, N .
\end{gathered}
$$

Proof. From (2.12), one can obtain

$$
\begin{array}{rlr}
-2 R_{00}=-e^{f_{u}} \frac{\partial f_{u}}{\partial u} 2 V, & -2 R_{0 i}=0, & -2 R_{0 N+1}=e^{f_{u}} \frac{\partial f_{u}}{\partial u}, \\
-2 R_{i k}=e^{f_{u}} \frac{\partial f_{u}}{\partial u} \delta_{i k}, & -2 R_{i N+1}=0, & -2 R_{N+1 N+1}=0,
\end{array}
$$

where $R_{\alpha \beta}$ are the Ricci curvatures with the metric $g_{u}$.

Then from the conformal Ricci curvatures obtained in Proposition 3.2, and through a direct computation, one can get the conclusion.

\section{Examples}

In this section, the conformal geometric structures of two famous classical Hamiltonian systems are shown. Assuming that $f: M \rightarrow \mathbb{R}$ ia a smooth function and the conformal metric $\tilde{g}=e^{f} g$, where $M$ is the configuration space and $g$ is the Eisenhart metric. For the following examples, the total energy $E$ is assumed to be a constant.

Example 4.1. For an $N$ degrees of freedom linear Hamiltonian system, the Hamiltonian is given by

$$
H=\frac{1}{2} \sum_{i=1}^{N} p_{i}^{2}+V\left(q^{1}, q^{2}, \ldots, q^{N}\right)
$$


in which

$$
V=\frac{1}{2} \sum_{i=1}^{N-1} \omega_{i}^{2}\left(q^{i}\right)^{2}-\frac{\lambda^{2}}{2}\left(q^{N}\right)^{2}
$$

where $\omega_{i}$ and $\lambda$ are constants.

This system consists of $N-1$ uncoupled linear oscillators, with the remaining uncoupled degree of freedom consisting of a parabolic barrier.

From (4.2), one can get

$$
\begin{gathered}
\nabla V=\left(\omega_{1}^{2} q^{1}, \omega_{2}^{2} q^{2}, \ldots, \omega_{N-1}^{2} q^{N-1},-\lambda^{2} q^{N}\right), \\
\Delta V=\sum_{i=1}^{N-1} \omega_{i}^{2}-\lambda^{2} .
\end{gathered}
$$

The coordinate of the manifold $M \times \mathbb{R}^{2}$ is $q=\left(q^{0}=t, q^{1}, q^{2}, \ldots, q^{N}, q^{N+1}\right)$ where $q^{N+1}=$ $(t / 2)+C_{2}^{2}-\int_{0}^{t} L \mathrm{~d} \tau$.

Proposition 4.2. The conformal Ricci curvatures and scalar curvature of the $N$ degrees of freedom linear Hamiltonian system are given by

$$
\begin{gathered}
\tilde{R}_{00}=\sum_{i=1}^{N-1} \omega_{i}^{2}-\lambda^{2}+V \Delta f+\frac{N}{2}(\nabla f \cdot \nabla V)+\frac{N}{2} V|\nabla f|^{2}, \quad \tilde{R}_{0 k}=0, \\
\tilde{R}_{0 N+1}=-\frac{1}{2} \Delta f-\frac{N}{4}|\nabla f|^{2}, \quad \widetilde{R}_{i N+1}=0, \quad \widetilde{R}_{N+1 N+1}=0, \\
\tilde{R}_{i k}=-\frac{N}{2} \partial_{i} \partial_{k} f+\frac{N}{4} \partial_{i} f \partial_{k} f-\delta_{i k}\left(\frac{1}{2} \Delta f+\frac{N}{4}|\nabla f|^{2}\right), \\
\tilde{R}=-e^{-f}\left((N+1) \Delta f+\frac{N(N+1)}{4}|\nabla f|^{2}\right),
\end{gathered}
$$

where $i, k$ are from 1 to $N$.

Moreover, from (3.12), we can get the geodesic equations as

$$
\begin{gathered}
q^{0}=t, \\
\frac{\mathrm{d}^{2} q^{k}}{\mathrm{~d} t^{2}}-\frac{1}{2} \partial_{k} f\left(\frac{\mathrm{d} q^{k}}{\mathrm{~d} t}\right)^{2}+\left(E-V+\frac{1}{2}\right) \partial_{k} f+\omega_{k}^{2} q^{k}=0, \quad k=1,2, \ldots, N-1, \\
\frac{\mathrm{d}^{2} q^{N}}{\mathrm{~d} t^{2}}-\frac{1}{2} \partial_{N} f\left(\frac{\mathrm{d} q^{N}}{\mathrm{~d} t}\right)^{2}+\left(E-V+\frac{1}{2}\right) \partial_{k} f-\lambda^{2} q^{N}=0, \\
q^{N+1}=\frac{t}{2}+C_{2}^{2}-\int_{0}^{t} L \mathrm{~d} \tau,
\end{gathered}
$$

where $L=E-2 V$, and $f$ satisfies $\mathrm{d} f / \mathrm{d} t=0$. 
International Journal of Mathematics and Mathematical Sciences

Remark 4.3. When $f$ is a constant function, (4.5) yields that

$$
\begin{gathered}
q^{0}=t, \\
\frac{\mathrm{d}^{2} q^{k}}{\mathrm{~d} t^{2}}+\omega_{k}^{2} q^{k}=0, \quad k=1,2, \ldots, N-1, \\
\frac{\mathrm{d}^{2} q^{N}}{\mathrm{~d} t^{2}}-\lambda^{2} q^{N}=0, \\
q^{N+1}=\frac{t}{2}+C_{2}^{2}-\int_{0}^{t} L \mathrm{~d} \tau .
\end{gathered}
$$

For the $N$ degrees of freedom linear Hamiltonian system, from the equations of flows in (3.21), we can get the following.

Proposition 4.4. The equations of flow are given by

$$
\begin{gathered}
e^{f_{u}} \frac{\partial f_{u}}{\partial u}=-\Delta f_{u}-\frac{N}{2}\left|\nabla f_{u}\right|^{2}, \\
\sum_{i=1}^{N-1}\left(\omega_{i}^{2} q^{i} \partial_{i} f_{u}\right)-\lambda^{2} q^{N} \partial_{N} f_{u}+\frac{2}{N}\left(\sum_{i=1}^{N-1}\left(\omega_{i}^{2}\right)-\lambda^{2}\right)=0, \\
2 \partial_{i} \partial_{k} f_{u}-\partial_{i} f_{u} \partial_{k} f_{u}=0, \quad i \neq k, \\
\Delta f_{u}-N \partial_{i} \partial_{i} f_{u}+\frac{N}{2}\left(\partial_{i} f_{u}\right)^{2}+\frac{N-1}{2}\left|\nabla f_{u}\right|^{2}=0, \quad i=1,2, \ldots, N,
\end{gathered}
$$

where $\left\{f_{u}\left|f_{u}\right|_{u=0}=0\right\}$ is a family of smooth functions.

Example 4.5. For the Hénon-Heiles Model, the Hamiltonian is

$$
H=\frac{1}{2}\left(p_{1}^{2}+p_{2}^{2}\right)+V\left(q^{1}, q^{2}\right)
$$

in which the potential $V$ is given by

$$
V=\frac{1}{2}\left(\left(q^{1}\right)^{2}+\left(q^{2}\right)^{2}\right)+\left(q^{1}\right)^{2} q_{2}-\frac{1}{3}\left(q^{2}\right)^{3}
$$

Next we are going to study the geometry of the Hénon-Heiles Model and obtain the numerical solutions. 
Proposition 4.6. The conformal Ricci curvatures and scalar curvature are given by

$$
\begin{gathered}
\widetilde{R}_{00}=\Delta V+V \Delta f+(\nabla f \cdot \nabla V)+V|\nabla f|^{2}, \quad \tilde{R}_{0 k}=0, \\
\widetilde{R}_{03}=-\frac{1}{2} \Delta f-\frac{1}{2}|\nabla f|^{2}, \quad \tilde{R}_{i 3}=0, \quad \widetilde{R}_{33}=0 \\
\tilde{R}_{i k}=-\partial_{i} \partial_{k} f+\frac{1}{2} \partial_{i} f \partial_{k} f-\delta_{i k}\left(\frac{1}{2} \Delta f+\frac{1}{2}|\nabla f|^{2}\right), \\
\tilde{R}=-e^{-f}\left(3 \Delta f+\frac{3}{2}|\nabla f|^{2}\right),
\end{gathered}
$$

where $i, k$ are from 1 to $2, \nabla V=\left(q^{1}+2 q^{1} q^{2}, q^{2}+\left(q^{1}\right)^{2}-\left(q^{2}\right)^{2}\right)$ and $\Delta V=2$.

From the Hamiltonian equations, one can get that

$$
\begin{aligned}
E-V & =H-V \\
& =\frac{1}{2}\left(p_{1}^{2}+p_{2}^{2}\right) \\
& =\frac{1}{2}\left(\left(\frac{\mathrm{d} q^{1}}{\mathrm{~d} t}\right)^{2}+\left(\frac{\mathrm{d} q^{2}}{\mathrm{~d} t}\right)^{2}\right) .
\end{aligned}
$$

Then the geodesic equations are given by

$$
\begin{gathered}
q^{0}=t, \\
\frac{\mathrm{d}^{2} q^{1}}{\mathrm{~d} t^{2}}-\frac{1}{2} \partial_{1} f\left(\frac{\mathrm{d} q^{1}}{\mathrm{~d} t}\right)^{2}+\left(E-V+\frac{1}{2}\right) \partial_{1} f+q^{1}+2 q^{1} q^{2}=0, \\
\frac{\mathrm{d}^{2} q^{2}}{\mathrm{~d} t^{2}}-\frac{1}{2} \partial_{2} f\left(\frac{\mathrm{d} q^{2}}{\mathrm{~d} t}\right)^{2}+\left(E-V+\frac{1}{2}\right) \partial_{2} f+q^{2}+\left(q^{1}\right)^{2}-\left(q^{2}\right)^{2}=0, \\
q^{3}=\frac{t}{2}+C_{2}^{2}-\int_{0}^{t} L \mathrm{~d} \tau,
\end{gathered}
$$

where $\mathrm{d} f / \mathrm{d} t=0$, and $E-V$ is given by (4.11). 


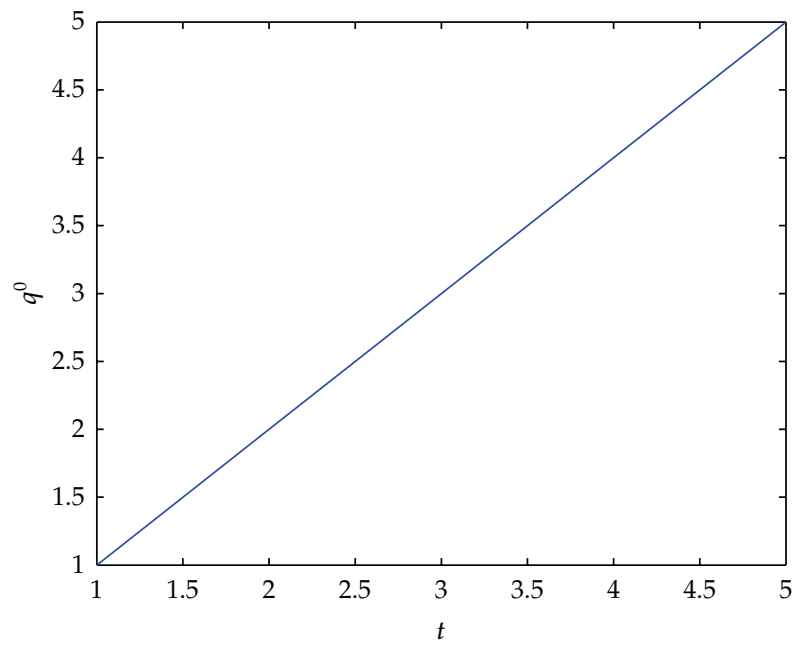

Figure 1: Component $q^{0}$ of the geodesic.

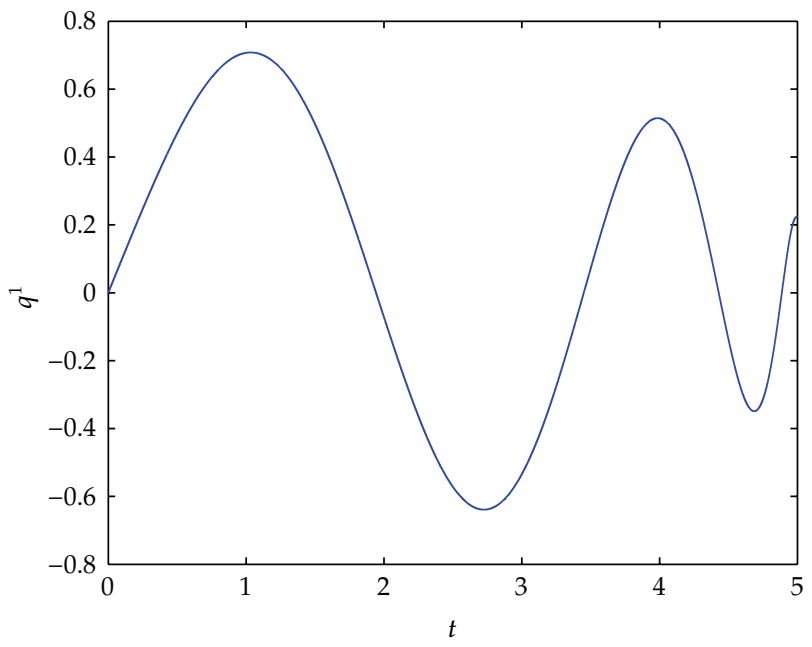

Figure 2: Component $q^{1}$ of the geodesic.

Remark 4.7. When $f$ is a constant function on $M$, the geodesic equations are given by

$$
\begin{gathered}
q^{0}=t \\
\frac{\mathrm{d}^{2} q^{1}}{\mathrm{~d} t^{2}}+q^{1}+2 q^{1} q^{2}=0, \\
\frac{\mathrm{d}^{2} q^{2}}{\mathrm{~d} t^{2}}+q^{2}+\left(q^{1}\right)^{2}-\left(q^{2}\right)^{2}=0, \\
q^{3}=\frac{t}{2}+C_{2}^{2}-\int_{0}^{t} L \mathrm{~d} \tau .
\end{gathered}
$$




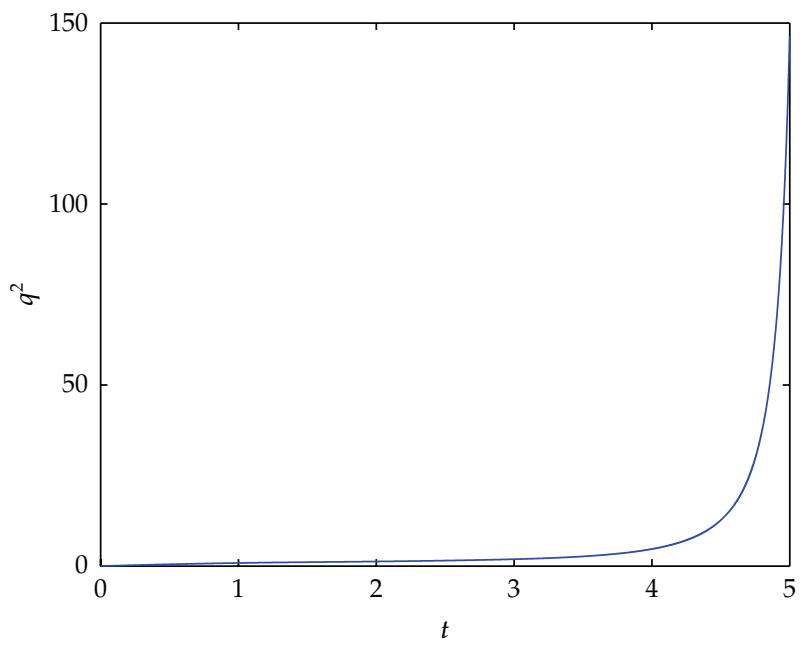

Figure 3: Component $q^{2}$ of the geodesic.

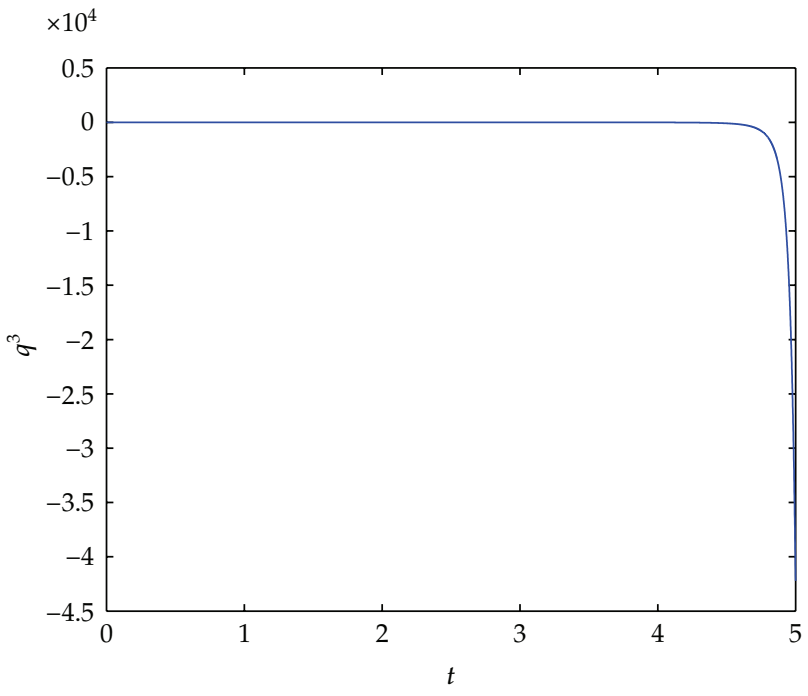

Figure 4: Component $q^{3}$ of the geodesic.

Moreover, for the above geodesic equations of the Hénon-Heiles model in (4.13) which can be reduced into the following first-order differential equations in (4.14), according to the theory of ordinary differential equations, we know that when the initial values are given, the solution of (4.13) uniquely exists.

Next we are going to study the solution of the above geodesic equations (4.13) through numerical simulations. In order to get the reduced equations and do simulation, firstly we introduce two parameters $p_{1}, p_{2}$, which are the generalized momenta, and $p_{i}=\mathrm{d} q^{i} / \mathrm{d} t, i=1,2$. 


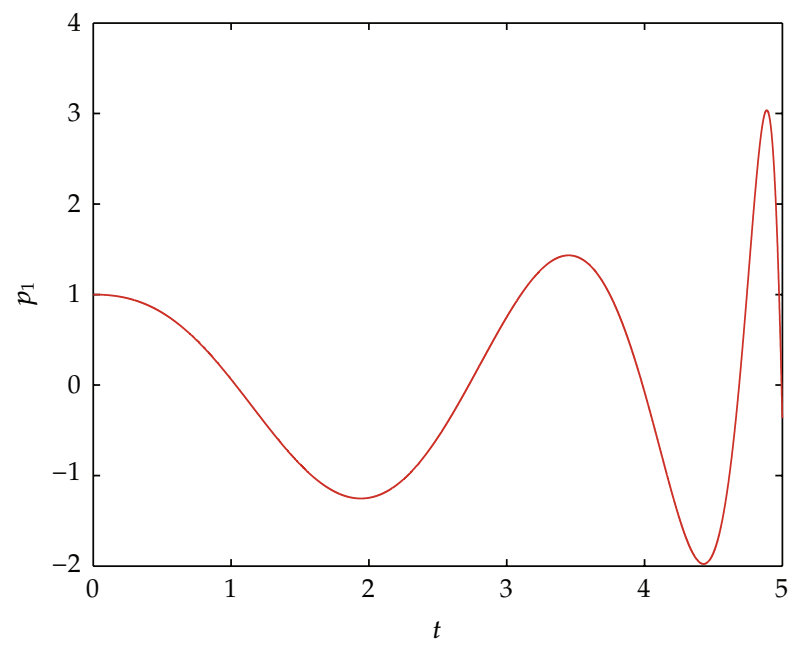

Figure 5: Generalized momentum $p_{1}$.

Then the second-order differential equations in (4.13) become into the following first-order differential equations

$$
\begin{gathered}
q^{0}=t \\
\frac{\mathrm{d} q^{1}}{\mathrm{~d} t}=p_{1}, \\
\frac{\mathrm{d} p_{1}}{\mathrm{~d} t}+q^{1}+2 q^{1} q^{2}=0, \\
\frac{\mathrm{d} q^{2}}{\mathrm{~d} t}=p_{2} \\
\frac{\mathrm{d} p_{2}}{\mathrm{~d} t}+q^{2}+\left(q^{1}\right)^{2}-\left(q^{2}\right)^{2}=0, \\
q^{3}=\frac{t}{2}+C_{2}^{2}-\int_{0}^{t} L \mathrm{~d} \tau,
\end{gathered}
$$

where $L=E-2 V$.

Let the initial vector $\left(q^{1}, q^{2}, q^{3}, p_{1}, p_{2}\right)$ be $(0,0,1,1,1)$, and the interval for time $t$ be $[0,5]$, by use of the ode45 method in Matlab, that is, Runge-Kutta $(4,5)$ method, we can get the following numerical results of geodesics and the generalized momenta (Figures 1,4).

Figures 2 and 3 above give the motion of the Hénon-Heiles model. Figures 5 and 6 describe the forms of generalized momenta. 


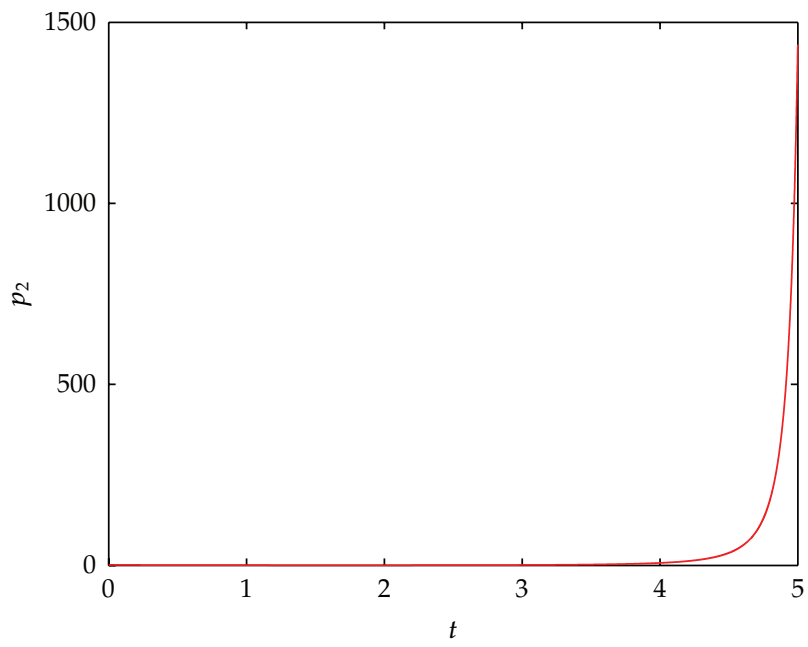

Figure 6: Generalized momentum $p_{2}$.

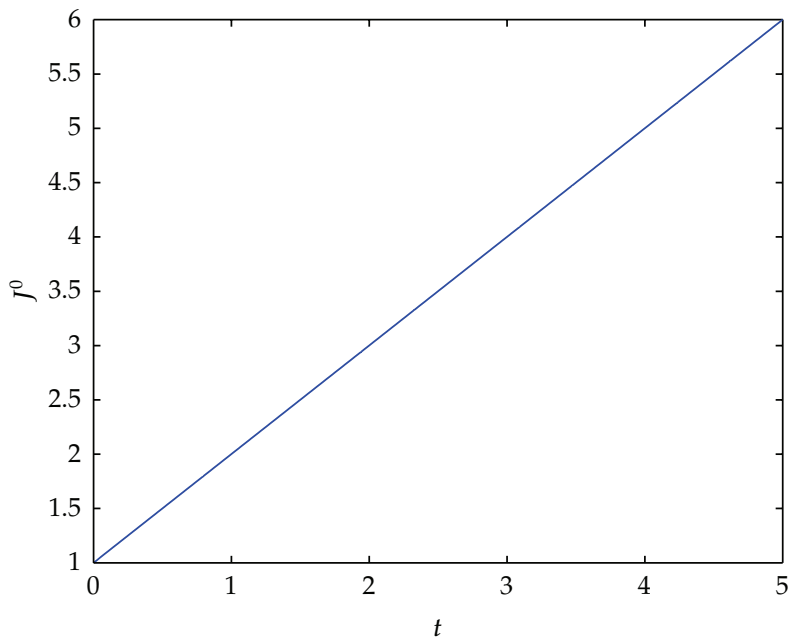

Figure 7: Component $J^{0}$ of the Jacobi field.

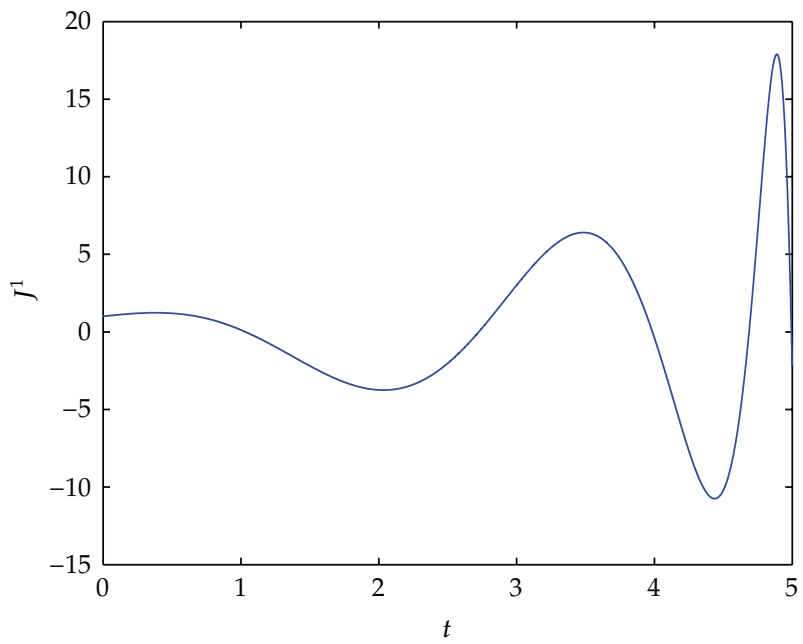

Figure 8: Component $J^{1}$ of the Jacobi field. 
International Journal of Mathematics and Mathematical Sciences

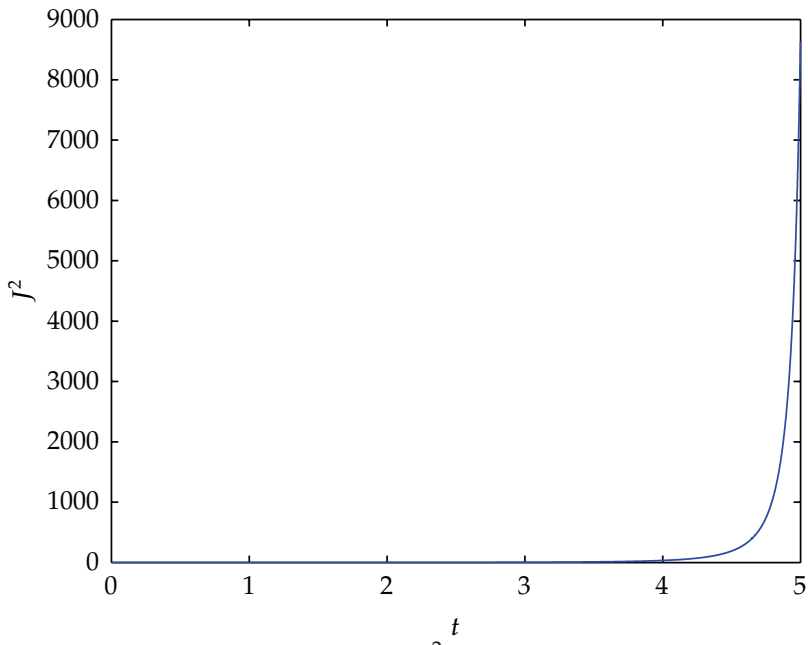

Figure 9: Component $J^{2}$ of the Jacobi field.

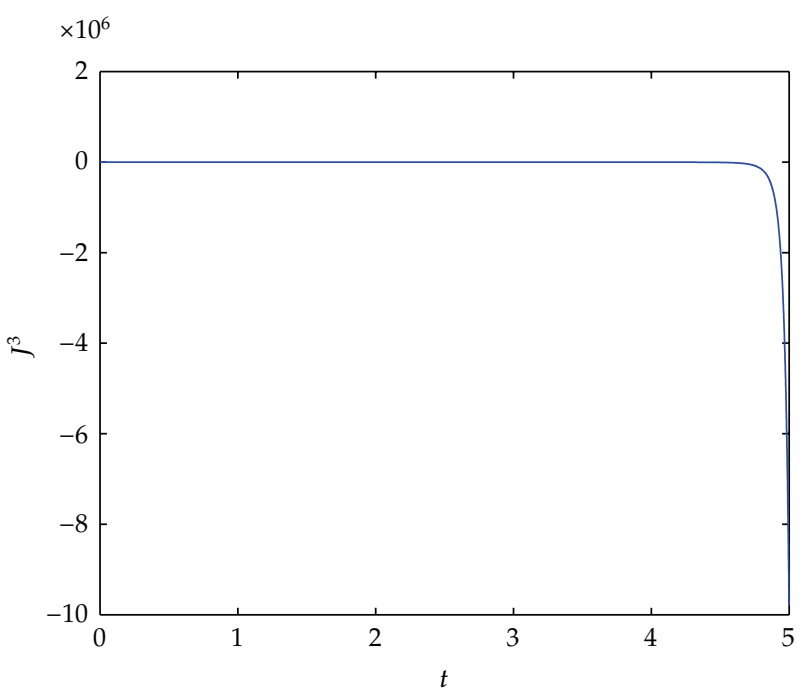

Figure 10: Component $J^{3}$ of the Jacobi field.

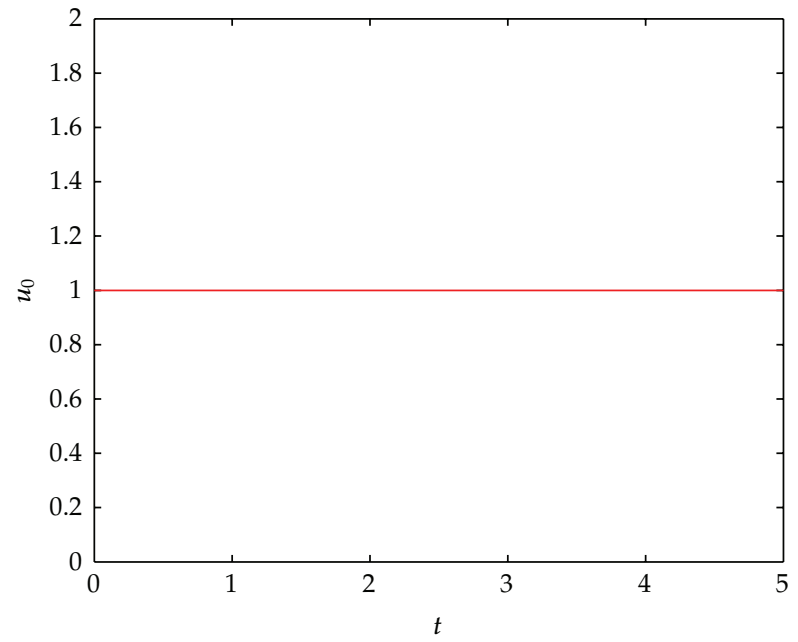

Figure 11: Component $u_{0}$. 


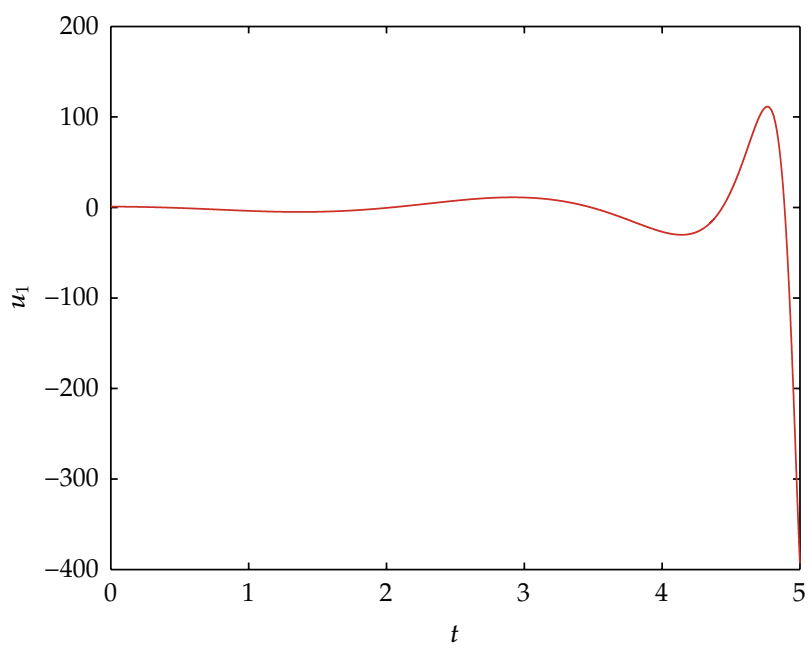

Figure 12: Component $u_{1}$.

Proposition 4.8. When $f$ is a constant function on $M$, the Jacobi field along the geodesic, which is the solution of (4.13), is given by

$$
\begin{aligned}
& \frac{\mathrm{d}^{2} J^{0}}{\mathrm{~d} t^{2}}=0 \\
& \frac{\mathrm{d}^{2} J^{1}}{\mathrm{~d} t^{2}}+2\left(q^{1}+2 q^{1} q^{2}\right) \frac{\mathrm{d} J^{0}}{\mathrm{~d} t}+\left(1+2 q^{2}\right) J^{1}+2 q^{1} J^{2}=0 \\
& \frac{\mathrm{d}^{2} J^{2}}{\mathrm{~d} t^{2}}+2\left(q^{2}+\left(q^{1}\right)^{2}-\left(q^{2}\right)^{2}\right) \frac{\mathrm{d} J^{0}}{\mathrm{~d} t}+2 q^{1} J^{1}+\left(1-2 q^{2}\right) J^{2}=0 \\
& \frac{\mathrm{d}^{2} J^{3}}{\mathrm{~d} t^{2}}-2 \frac{\mathrm{d} V}{\mathrm{~d} t} \frac{\mathrm{d} J^{0}}{\mathrm{~d} t}-2\left(q^{1}+2 q^{1} q^{2}\right) \frac{\mathrm{d} J^{1}}{\mathrm{~d} t}-2\left(q^{2}+\left(q^{1}\right)^{2}-\left(q^{2}\right)^{2}\right) \frac{\mathrm{d} J^{2}}{\mathrm{~d} t}-|\nabla V|^{2} J^{0} \\
& \quad-2\left(\left(1+2 q^{2}\right) \frac{\mathrm{d} q^{1}}{\mathrm{~d} t}+2 q^{1} \frac{\mathrm{d} q^{2}}{\mathrm{~d} t}\right) J^{1}-2\left(2 q^{1} \frac{\mathrm{d} q^{1}}{\mathrm{~d} t}+\left(1-2 q^{2}\right) \frac{\mathrm{d} q^{2}}{\mathrm{~d} t}\right) J^{2}=0
\end{aligned}
$$

where $\mathrm{d} V / \mathrm{d} t=\left(q^{1}+2 q^{1} q^{2}\right)\left(\mathrm{d} q^{1} / \mathrm{d} t\right)+\left(q^{2}+\left(q^{1}\right)^{2}-\left(q^{2}\right)^{2}\right)\left(\mathrm{d} q^{2} / \mathrm{d} t\right)$, and $|\nabla V|^{2}=\left(q^{1}+2 q^{1} q^{2}\right)^{2}+$ $\left(q^{2}+\left(q^{1}\right)^{2}-\left(q^{2}\right)^{2}\right)^{2}$.

Moreover, the Jacobi equations in (4.15) are also ordinary differential equations which can be reduced into first-order differential equations, and when the initial values are given, the solution of (4.15) uniquely exists. 


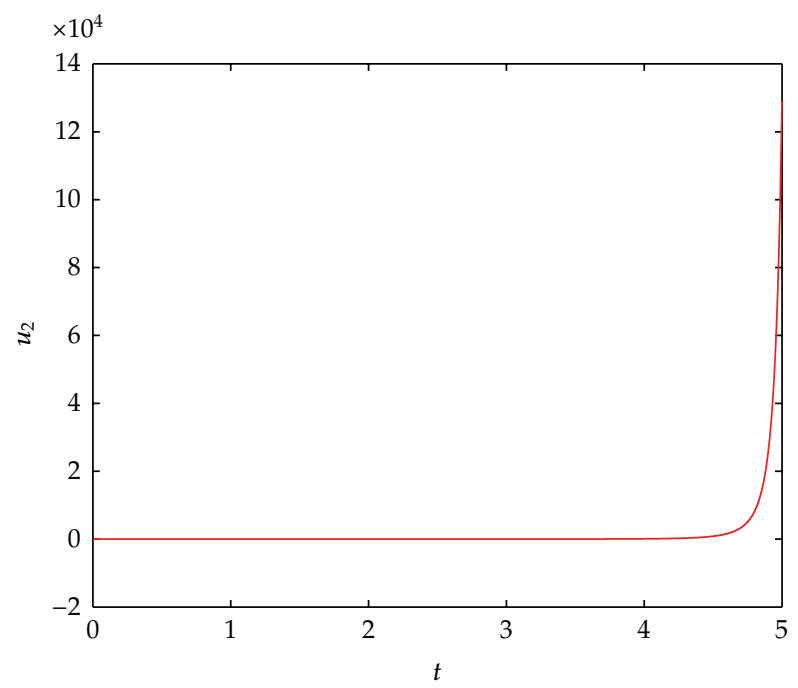

Figure 13: Component $u_{2}$.

For (4.15), next we are going to find the numerical solution of the Jacobi field. Using the similar process as above, we introduce four parameters $u_{0}, u_{1}, \ldots, u_{3}$ and assume that $u_{i}=\mathrm{d} J^{i} / \mathrm{d} t, i=0,1, \ldots, 3$. Then (4.15) becomes into

$$
\begin{aligned}
& \frac{\mathrm{d} J^{0}}{\mathrm{~d} t}=u_{0}, \\
& \frac{\mathrm{d} u_{0}}{\mathrm{~d} t}=0, \\
& \frac{\mathrm{d} J^{1}}{\mathrm{~d} t}=u_{1}, \\
& \frac{\mathrm{d} u_{1}}{\mathrm{~d} t}+2\left(q^{1}+2 q^{1} q^{2}\right) u_{0}+\left(1+2 q^{2}\right) J^{1}+2 q^{1} J^{2}=0, \\
& \frac{\mathrm{d} J^{2}}{\mathrm{~d} t}=u_{2}, \\
& \frac{\mathrm{d} u_{2}}{\mathrm{~d} t}+2\left(q^{2}+\left(q^{1}\right)^{2}-\left(q^{2}\right)^{2}\right) u_{0}+2 q^{1} J^{1}+\left(1-2 q^{2}\right) J^{2}=0, \\
& \frac{\mathrm{d} J^{3}}{\mathrm{~d} t}=u_{3}, \\
& \frac{\mathrm{d} u_{3}}{\mathrm{~d} t}-2 \frac{\mathrm{d} V}{\mathrm{~d} t} u_{0}-2\left(q^{1}+2 q^{1} q^{2}\right) u_{1}-2\left(q^{2}+\left(q^{1}\right)^{2}-\left(q^{2}\right)^{2}\right) u_{2}-|\nabla V|^{2} J^{0} \\
& \quad-2\left(\left(1+2 q^{2}\right) p_{1}+2 q^{1} p_{2}\right) J^{1}-2\left(2 q^{1} p_{1}+\left(1-2 q^{2}\right) p_{2}\right) J^{2}=0,
\end{aligned}
$$

where $\mathrm{d} q^{i} / \mathrm{d} t$ and $\mathrm{d}^{2} q^{i} / \mathrm{d} t^{2}$ are replaced by $p_{i}$ and $\mathrm{d} p_{i} / \mathrm{d} t$ given in (4.14), respectively. 


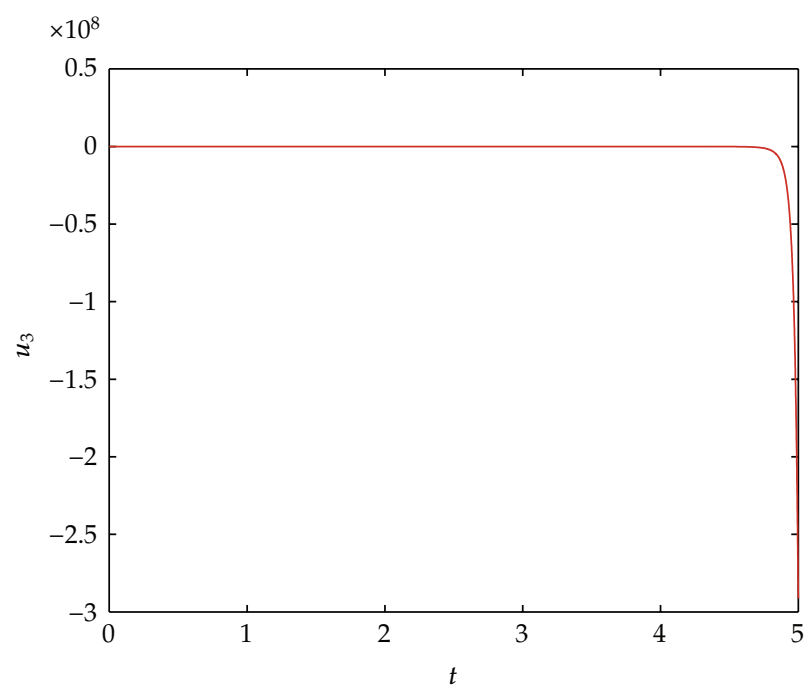

Figure 14: Component $u_{3}$.

We assume that the initial vector $\left(J^{0}, J^{1}, J^{2}, J^{3}, u_{0}, u_{1}, u_{2}, u_{3}\right)$ is $(1,1,1,1,1,1,1,1)$, and the time interval is $[0,5]$. Also using the ode45 method in Matlab, and the first-order differential equations of geodesics and generalized momenta in (4.14), we can get the following numerical results of Jacobi field.

Figures $7,8,9$, and 10 show us that the Jacobi field is divergent when the time $t \rightarrow+\infty$, which implies the instability of the geodesic spread. For more please refer to [21, 22] about the instability of the geodesic derivation equation, that is, the Jacobi equation for the HénonHeiles model, and $[16,17]$ about the geodesic spread and geodesic derivative equation and their applications (Figures 11-14).

Proposition 4.9. For the Hénon-Heiles model, from Theorem 3.11, we can obtain the equations of flow as

$$
\begin{gathered}
e^{f_{u}} \frac{\partial f_{u}}{\partial u}=-\Delta f_{u}-\left|\nabla f_{u}\right|^{2}, \\
\left(q^{1}+2 q^{1} q^{2}\right) \partial_{1} f_{u}+\left(q^{2}+\left(q^{1}\right)^{2}-\left(q^{2}\right)^{2}\right) \partial_{2} f_{u}=-2, \\
2 \partial_{i} \partial_{k} f_{u}-\partial_{i} f_{u} \partial_{k} f_{u}=0, \quad i \neq k, \\
\Delta f_{u}-2 \partial_{i} \partial_{i} f_{u}+\left(\partial_{i} f_{u}\right)^{2}+\frac{1}{2}\left|\nabla f_{u}\right|^{2}=0, \quad i=1,2 .
\end{gathered}
$$

\section{Conclusion}

The theory of conformal metric in Riemannian geometry is applied to characterize the geometry of the classical Hamiltonian dynamics with the conformal Eisenhart metric (Section 3). We obtain the Ricci curvatures, the scalar curvatures, the geodesic equations, the Jacobi equations, and the equation of a certain flow. The relation between the curvatures 
with Eisenhart metric and its conformal metric can also be shown clearly. As in the examples, the conformal curvatures, the equation of a certain flow, and the numerical solutions of the geodesics, generalized momenta, and Jacobi field along the geodesics are obtained (Section 4).

\section{Acknowledgment}

This paper is supported by the National Natural Science Foundations of China (no. 10871218, no. 10932002).

\section{References}

[1] L. Casetti, M. Pettini, and E. G. D. Cohen, "Geometric approach to Hamiltonian dynamics and statistical mechanics," Physics Reports A, vol. 337, no. 3, pp. 237-341, 2000.

[2] A. Yahalom, J. Levitan, M. Lewkowicz, and L. Horwitz, "Lyapunov vs. geometrical stability analysis of the Kepler and the restricted three body problems," Physics Letters A, vol. 375, no. 21, pp. 2111-2117, 2011.

[3] T. Iwai and H. Yamaoka, "Stratified dynamical systems and their boundary behaviour for three bodies in space, with insight into small vibrations," Journal of Physics A, vol. 38, no. 25, pp. 5709-5730, 2005.

[4] G. Blankenstein, "A joined geometric structure for hamiltonian and gradient control systems," in Lagrangian and Hamiltonian Methods for Nonlinear Control 2003, pp. 51-56, IFAC, Laxenburg, 2003.

[5] E. V. Ferapontov, "Differential geometry of nonlocal Hamiltonian operators of hydrodynamic type," Funktsional Analiz i ego Prilozheniya, vol. 25, no. 3, pp. 37-49, 1991.

[6] E. V. Ferapontov, "Hamiltonian systems of hydrodynamic type and their realizations on hypersurfaces of a pseudo-Euclidean space," in Problems in Geometry, vol. 22 of Itogi Nauki i Tekhniki, pp. 59-96, Akad. Nauk SSSR Vsesoyuz. Inst. Nauchn. i Tekhn. Inform., Moscow, Russia, 1990.

[7] T. Kambe, Geometrical Theory of Dynamical Systems and Fluid Flows, vol. 23 of Advanced Series in Nonlinear Dynamics, World Scientific, Hackensack, NJ, USA, 2005.

[8] S. Tanimura and T. Iwai, "Reduction of quantum systems on Riemannian manifolds with symmetry and application to molecular mechanics," Journal of Mathematical Physics, vol. 41, no. 4, pp. 1814-1842, 2000.

[9] T. Uzer, C. Jaffé, J. Palacián, P. Yanguas, and S. Wiggins, "The geometry of reaction dynamics," Nonlinearity, vol. 15, no. 4, pp. 957-992, 2002.

[10] A. Yu. Boldin, A. A. Bronnikov, V. V. Dmitrieva, and R. A. Sharipov, "Complete normality conditions for dynamical systems on riemannian manifolds," Rossiǔskaya Akademiya Nauk. Teoreticheskaya $i$ Matematicheskaya Fizika, vol. 103, no. 2, pp. 267-275, 1995.

[11] P. Boyland and C. Golé, "Lagrangian systems on hyperbolic manifolds," Ergodic Theory and Dynamical Systems, vol. 19, no. 5, pp. 1157-1173, 1999.

[12] R. A. Sharipov, "Newtonian normal shift in multidimensional Riemannian geometry," Matematicheskiป Sbornik, vol. 192, no. 6, pp. 105-144, 2001.

[13] M. P. do Carmo, Riemannian Geometry, Mathematics: Theory \& Applications, Birkhäuser, Boston, Mass, USA, 1992.

[14] P. Petersen, Riemannian Geometry, vol. 171 of Graduate Texts in Mathematics, Springer, New York, NY, USA, 2nd edition, 2006.

[15] L. Todjihounde, "Ricci deformation and conformal change of metrics," IC /99/89, International Centre for Theoretical Physics, Trieste, Italy, 1999.

[16] L. Peng, H. Sun, D. Sun, and J. Yi, "The geometric structures and instability of entropic dynamical models," Advances in Mathematics, vol. 227, no. 1, pp. 459-471, 2011.

[17] C. W. Misner, K. S. Thorne, and J. A. Wheeler, Gravitation, W. H. Freeman and Co., San Francisco, Calif, USA, 1973.

[18] R. S. Hamilton, "Three-manifolds with positive Ricci curvature," Journal of Differential Geometry, vol. 17, no. 2, pp. 255-306, 1982.

[19] J. E. Marsden and T. S. Ratiu, Introduction to Mechanics and Symmetry: A Basic Exposition of Classical Mechanical Systems, vol. 17 of Texts in Applied Mathematics, Springer, New York, NY, USA, 1994. 
[20] L. P. Eisenhart, "Dynamical trajectories and geodesics," Annals of Mathematics(Princeton), vol. 30, pp. 591-606, 1929.

[21] V. G. Gurzadyan, "Chaotic phenomena in astrophysics and cosmology," in Proceedings of the 10th Brazilian School of Cosmology and Gravitation, July-August 2002.

[22] H. E. Kandrup, "Geometric interpretation of chaos in two-dimensional hamiltonian systems," Physical Review E, vol. 57, no. 3, part A, pp. 2722-2732, 1997. 


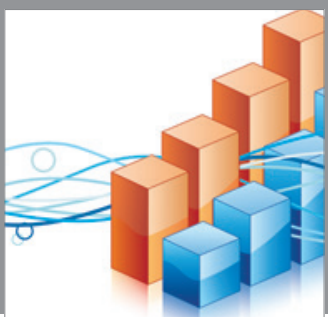

Advances in

Operations Research

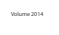

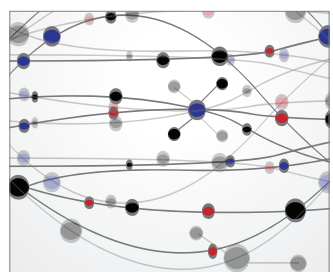

\section{The Scientific} World Journal
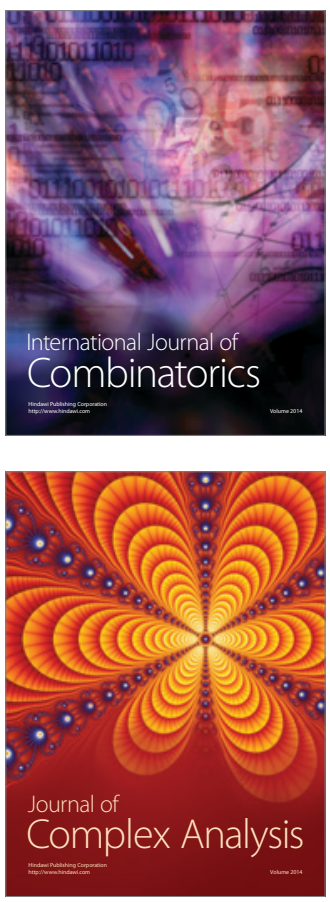

International Journal of

Mathematics and

Mathematical

Sciences
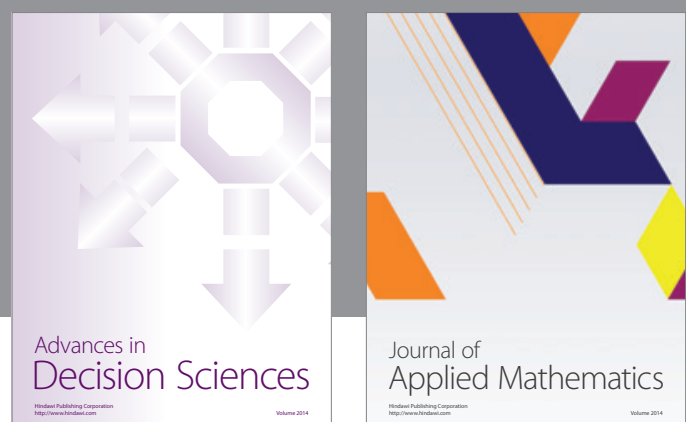

Journal of

Applied Mathematics
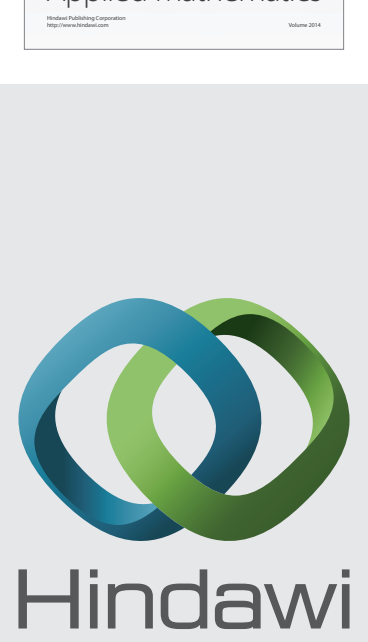

Submit your manuscripts at http://www.hindawi.com
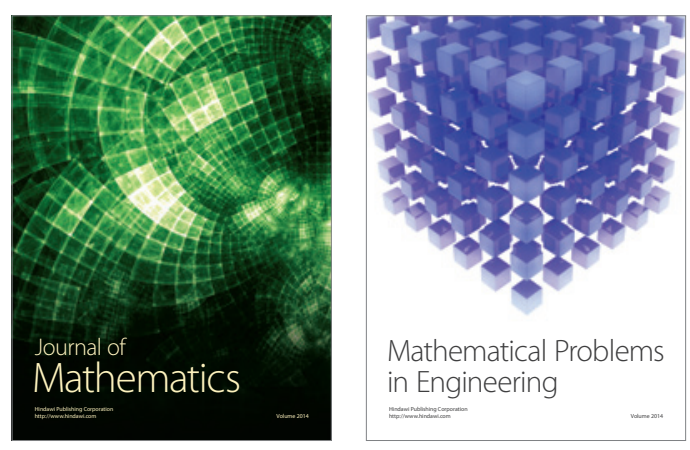

Mathematical Problems in Engineering
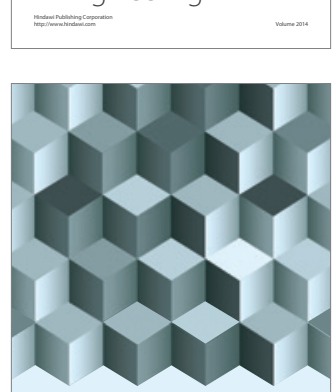

Journal of

Function Spaces
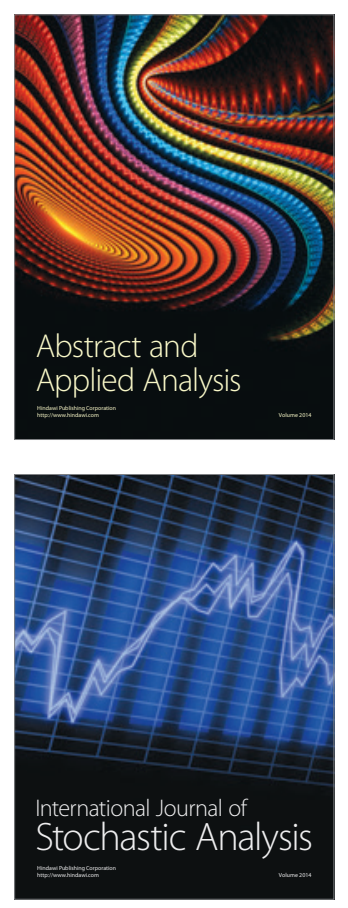

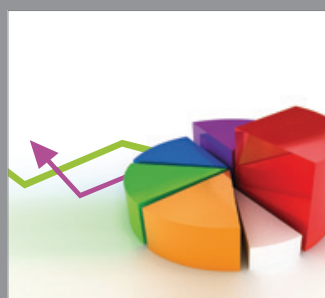

ournal of

Probability and Statistics

Promensencen
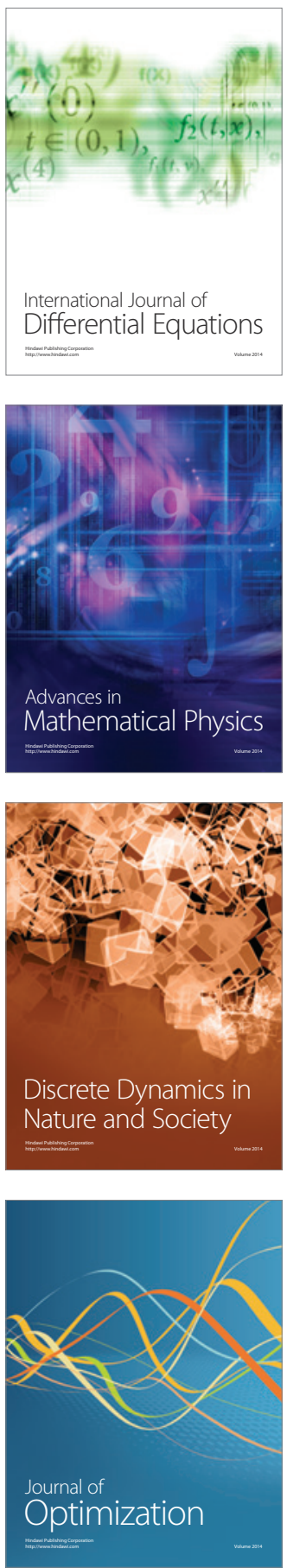